\title{
Special Issue on: Cooperative and Distributed Wireless Communications
}

\author{
Abbas Jamalipour • Ian Oppermann • \\ Nirwan Ansari · Graeme Woodward
}

Received: 29 July 2013/ Accepted: 31 July 2013/Published online: 7 August 2013

(C) Springer Science+Business Media New York 2013

Recent advances in radio technology as well as in hardware and software design have resulted in new possibilities for future mobile communication networks. Among those, cooperative communications among the peer nodes of a cellular network could create much anticipated improvement in coverage and capacity of the cellular systems. Such cooperation would generate a distributed network topology where the abundant processing power and storage capacity of the nodes can be shared for carrying out complex tasks. Distributed wireless communications would also enable mobile computing within the mobile cloud. In this context, the 23rd Annual Symposium on Personal Indoor and Mobile Radio Communications (PIMRC 2012) served as a major international telecommunication forum for researchers and technologists to discuss original contributions and results in all fields of personal, indoor and mobile communication systems, including those related to the above topic of cooperative and distributed wireless communications.

This special issue is based upon a selection of a very limited number of papers presented at IEEE PIMRC2012. Authors of the top 15 papers from the symposium were invited to submit an extended version of their papers to this special issue. Among those, we are happy to present you

\author{
A. Jamalipour $(\bowtie)$ \\ University of Sydney, Sydney, NSW, Australia \\ e-mail: a.jamalipour@ieee.org \\ I. Oppermann \\ CSIRO, Marsfield, NSW, Australia \\ N. Ansari \\ New Jersey Institute of Technology, Newark, NJ, USA \\ G. Woodward \\ University of Canterbury, Christchurch, New Zealand
}

with four papers that have been accepted after a rigorous peer review process.

The first paper, New Diversity Combining Receivers for Cooperative Multiplexing in Wireless Multiuser Relay Networks, by Chang Kyung Sung, Iain B. Collings, Maged Elkashlan, and Phee Lep Yeoh, proposes two new diversity combining receivers that support cooperative multiplexing in two-hop wireless multiuser relay networks. Cooperative multiplexing has the potential to double the achievable throughput by allowing the base station (BS) and the relay station (RS) to transmit to different users at the same time in the second time slot of the half time division duplexed (TDD) relay transmission. This throughput improvement comes at a cost of performance degradation due to interuser interference between the BS and the RS. To overcome this degradation, two new receivers for the relay-link users are proposed: (1) cooperative multiplexing optimum combining (CMOC) and (2) cooperative multiplexing selection combining (CMSC). Exact closed-form expressions are derived for the moment generation function, probability density function, and the cumulative distribution function of the output SINR, and new analytical expressions for the outage probability, symbol error rate, and achievable throughput are presented. The authors show that the symbol error rate performance and achievable throughput were notably improved relative to the standard single-channel receiver in the high interference regime.

The second paper, Improving Cooperative Transmission Feasibility by Network Reconfiguration in Limited Backhaul Networks, by Martin Draxler, Thorsten Biermann, and Holger Karl, develops a mixed integer linear program (MILP) and a BS selection heuristic for Coordinated MultiPoint (CoMP) that takes into account both aspects of the wireless channels and the backhaul network status. This heuristic can also identify which bottlenecks in the 
backhaul network make a particular BS selection infeasible. They exploit this to dynamically reconfigure the backhaul network according to the wireless requirements. Simulations show that the heuristic's solution quality is close to the optimum while execution time and memory consumption are reduced by multiple orders of magnitude compared to solving the problem via mathematical optimization. In addition, simulation results for network reconfiguration in different backhaul network scenarios illustrate how the proposed approach helps to better exploit available backhaul resources.

The third paper, A Novel Gateway Selection Technique for Throughput Optimization in Configurable Wireless Mesh Networks, by Wei Liu, Hiroki Nishiyama, Nei Kato, Yoshitaka Shimizu, and Tomoaki Kumagai, considers wireless mesh networks (WMNs) for the disaster recovery applications. WMN advantages including low up-front cost, easy network deployment, stable topology, robustness and reliable coverage. They can be utilized to restore network collapse after a disaster event. In this paper, based upon a new network infrastructure for WMNs to guarantee high network performance, the authors focus on the issue of throughput optimization to improve the performance for WMNs. Motivated by the observation that selecting a different mesh router as the gateway will lead to different network throughput capacity, they propose a novel selection technique to rapidly choose the optimal mesh router as the gateway. In addition, they take into account the traffic distribution for the mesh router to eliminate traffic congestion. Performance of the proposed method is evaluated by both numerical and simulated analysis and results demonstrate that the gateway selection method is effective to optimize the throughput for WMNs.

In the fourth and final paper, Performance Analysis of Communication System with Nonlinear Power Amplifier, by Ilia Iofedov, Igor Gutman, and Dov Wulich, a communication system with nonlinear power amplifier (PA) is considered. The PA nonlinearity forces the designer of a communication system to choose proper back-off, which influences three major parameters of the communication system: (i) power efficiency, (ii) adjacent channel power ratio (ACPR), and (iii) bit rate expressed by mutual information (MI). Given these three parameters, the authors evaluate the performance of a communication system with such a nonlinear PA using the fact that ACPR is dictated by the regulator/communication standard. Power efficiency and MI are computed as functions of ACPR. To illustrate the usefulness of the proposed method, the solid state power amplifier (SSPA), and two linearized versions of SSPA are considered, including a novel feed-forward approach. The analysis demonstrates that linearization improves the overall performance of communication systems.

We sincerely hope that this Special Issue will be enjoyable for all to read. The quality of this special issue depends upon the quality of articles and the stringent refereeing carried out by a number of volunteers. As Guest Editors, we wish to thank these referees whose helpful remarks have contributed to the final quality of the published papers, and the authors who have revised their articles within the tight deadlines, and have agreed to publish their work in this special issue of the Journal of Wireless Information Networks. We would also like to thank the Editor in Chief, Kaveh Pahlavan, for his support, his encouragement, and his guidance through this entire project.

\section{Author Biographies}

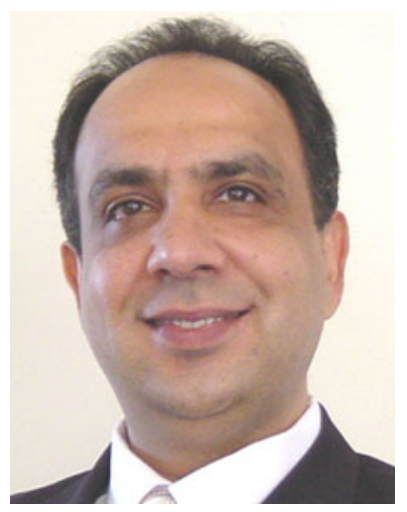

Abbas Jamalipour $\mathrm{He}$ is a Fellow of IEEE, IEICE, and Engineers Australia, a Professional Member of ACM, and an IEEE Distinguished Lecturer. He holds a Ph.D. in Electrical Engineering from Nagoya University, Japan. He is the Professor of Ubiquitous Mobile Networking, Deputy Director for the Centre of Excellence in Telecommunications, and leads the Wireless Networking Group (WiNG) at the University of Sydney, Australia. He is the author of six technical books, nine book chapters, and over 350 technical papers in scholarly journals and international conferences, as well as four patents, all in the area of wireless networking. He is the recipient of many prestigious awards including the 2010 IEEE ComSoc Harold Sobol Award, the 2010 Royal Academy of Engineering UK Distinguished Fellowship, the 2006 IEEE ComSoc Distinguished Contribution to Satellite Communications Award, the 2006 IEEE ComSoc Best Tutorial Paper Award, and ten best paper awards. He was the Editor-in-Chief IEEE Wireless Communications and currently he is an editor for several scholarly journals. He has served in many IEEE positions including ComSoc Vice President for Conferences; Member ComSoc Finance Committee; Member ComSoc On-Line Contents Committee; Member ComSoc Education Board; Member ComSoc Conference Boards; Member IEEE TAB/PSPB Products \& Services Committee; Chair Communication Switching and Routing TC; Chair Satellite and Space Communications TC; Vice Director Asia Pacific Board. He has been a General Chair/Technical Program Chair/Vice Chair of major IEEE conferences (e.g., RWS'08, RWS'09, WCNC'10, GLOBECOM'10, ICC'11, GLOBECOM'12, PIMRC'12; ICC'14). 


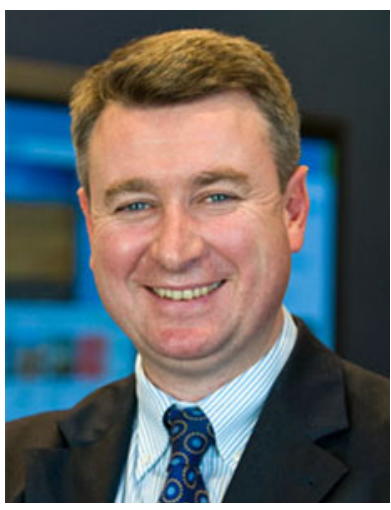

Ian Oppermann He heads up Australia's leading multidisciplinary ICT and services research activity, targeting productivity growth in Australia through frontier services innovation and by unlocking the value of a national broadband infrastructure. As Director of the Digital Productivity and Services Flagship at CSIRO, Dr Oppermann oversees ICT and services research in Government and Commercial Services, Smart Secure Infrastructure, and Health Services. His research interests include: signal Processing and decision systems, large scale, distributed information systems, radio channel measurement and modeling, location and positioning systems, radio communication systems including code division multiple access (CDMA) and Ultra wideband (UWB) systems, and network optimization. He is Chairman of the Executive Committee of the IEEE International Conference on Ultra Wideband (ICUWB), a position he has held since 2004, and was most recently the chairman of the IEEE Personal Indoor and Mobile Radio Communications Conference (PIMRC) in 2012. He has contributed to 6 books as editor or chapter author, authored or co-authored approximately 30 journal and 90 conference papers. Dr Oppermann has a Doctor of Philosophy in electrical engineering from Sydney University and an MBA from the University of London. He is a Fellow of the Institute of Engineers Australia, a Fellow of the IEEE and a member of the Australian Institute of Company Directors.

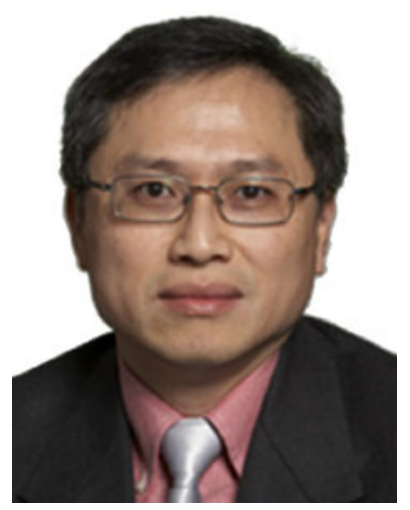

Nirwan Ansari He received the B.S.E.E. from NJIT, Newark, the M.S.E.E. from University of Michigan, Ann Arbor, and the $\mathrm{Ph} . \mathrm{D}$. degree from Purdue University, West Lafayette, IN, respectively. He is Professor of Electrical \& Computer Engineering at NJIT. He was Visiting (Chair) Professor at several universities. He authored Media Access Control and Resource Allocation (2013) with J. Zhang, and Computational Intelligence for Optimization (1997) with E.S.H. Hou, and edited Neural Networks in Telecommunications (1994) with B. Yuhas. His current research focuses on various aspects of broadband networks and multimedia communications. He has contributed over 450 technical papers, over one-third in widely cited refereed journals/magazines. He has guest edited a number of special issues, covering various emerging topics in communications and networking. He has served on the Editorial Board and Advisory Board of eight journals, and was elected to serve in the IEEE Communications Society (ComSoc) Board of Governors as a member-at-large (2013-2015). He has chaired ComSoc technical committees, and has been actively organizing numerous IEEE International Conferences/Symposia/Workshops, assuming various leadership roles. Some of his recognitions include IEEE Fellow, several Excellence in Teaching Awards, a couple of best paper awards, Thomas Alva Edison Patent Award (2010), NJ Inventors Hall of Fame Inventor of the Year Award (2012), Outstanding Service Recognition Award (2013 from ComSoc AHSN TC), and designation as an IEEE Communications Society Distinguished Lecturer (2006-2009, two terms). He was also awarded more than 20 US patents.

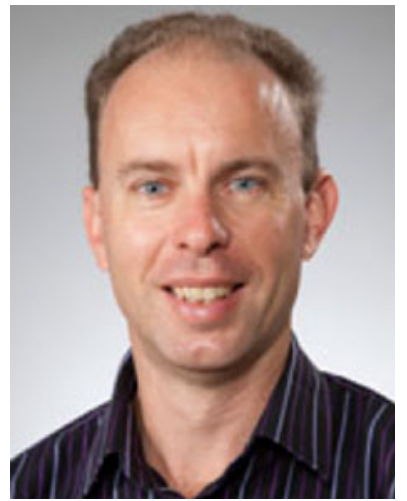

Graeme Woodward He joined the University of Canterbury in January 2011 as Research Leader of the Wireless Research Centre. Prior to joining UC he was Research Manager in the Telecommunications Research Laboratory, Toshiba Research Europe working closely with the University of Bristol and participating in numerous collaborative projects within the UK \& European research communities. Graeme has extensive industrial research experience covering mobile phone standards. He joined Bell Laboratories Research Sydney (Lucent Technologies) in 2001 to research 3G systems, multiuser detection, interference suppression, multi-antenna systems and adaptive digital filter algorithms for equalisation. He was part of the team which pioneered VLSI (Very Large Scale Integration) designs for MIMO (Multi-in/Multi-out antenna) reception of HSDPA (High Speed Downlink Packet Access). With the Bell Labs group transitioning to Agere Systems Australia and subsequently LSI Logic in 2003 and 2007 respectively, the focus moved to terminalside algorithms for HSDPA and long term evolution (LTE) with an increased emphasis on low power design. His earlier experience includes digital switching systems with Alcatel Australia and channel modelling, and multiuser detection for CDMA (code division multiple access) systems, with SP Communications in collaboration with The University of Oulu, Finland. Graeme was awarded B.Sc and BE and Ph.D. degrees from The University of Sydney in 1990, 1992 and 1999 respectively. Dr Woodward has authored ten journal articles, more than 30 conference papers and is an inventor on 12 US patent. He has served on several conference committees, including TPC chair for AusCTW05 and 06; local organising chair for ISSSTA04 and conference chair of the NZ Wireless Workshop 2011. He is a member of APESMA (The Association of Professional Engineers, Scientists and Managers Australia) and a senior member of the IEEE. 